\title{
Review of: "In-hospital mortality among immunosuppressed patients with COVID-19: Analysis from a national cohort in Spain"
}

\author{
Antonia Anna Lukito, Indah Sukmawati
}

Potential competing interests: The author(s) declared that no potential competing interests exist.

This was a retrospective cohort study based on a large multicenter registry in Spain with data from around 3 months span (March-June 2020) which was during the first COVID-19 wave. The first wave reached its peak in Spain on early April 2020 with 8000 new daily cases. ${ }^{1}$ It was not stated how many patients were in the registry during the course of the study, but it is estimated that it would reach around 150.000 confirmed cases during that period of time based on WHO data. ${ }^{2}$ In this study, the authors had included 13.206 patients with complete information which didn't reach $10 \%$ of the estimated total confirmed patients albeit the authors claim that this was the largest number of immunosuppressed patients published data to date. Hence, we do need to interpret the data carefully.

It is hypothesized that IS-patients would have higher in-hospital mortality and worse prognosis altogether which were the results that we found on this study. Interestingly, these presumably vulnerable group of patients were significantly less likely to be admitted to the ICU than non-IS patients. The authors stated that the reasons for these horrific decisions of choosing the ones that have better chance of survival get the ventilators and be admitted to the ICU, were because the overloaded healthcare system. Since the IS patients had an average of $60 \%$ higher chance of in-hospital death compared to non-IS patients, the IS patients never stood a chance battling COVID-19 at that point of time. A harsh fact and tremendously difficult decisions made by our dedicated and courageous colleagues in Spain. Yet, this might not be the only reason why the IS-patients have higher in-hospital mortality rate because even when compared amongst patients that were admitted to the ICU, the IS-patients still had higher mortality than its counterpart. The authors have rigorously analyzed in detailed statistics and adjusted them for confounders such as steroid use and other non-measured variables. One must keep in mind that this large multicenter data was gathered in time of global pandemic when Spain was one of the first country to take the hardest hit due to it large population of older age patients and an overwhelmed healthcare system, so it is understandable that some if not most of the data submitted to the registry might not be complete.

There are many variables to define an immunosuppressive patient. Even patients having the same type of cancer might have different immunological status depending on several things like age, stage of cancer, 
type of chemo, performance status, etc. There are other conditions that may cause a patient immune system suppressed that were beyond the scope of the authors' analysis due to lack of complete data such as multiple myeloma, myelodysplastic syndromes, advanced HIV infection, primary immunodeficiency, asplenia. All of this have been mentioned by the authors as limitations to their study.

In light of more understanding of the COVID-19 pathophysiology, public health protocol implementation and global vaccination efforts, the in-hospital situation nowadays might not be as chaotic as it was at the beginning of the pandemic. It would be very enlightening as we learn from the latest data on this topic with more confounders eliminated and more complete data submitted to the registry.

The interesting point to note in this study is the patients with prior routine steroids consumption have a higher mortality than their counterparts, since dexamethasone has been known as a recommended drug in severe COVID-19 patients. Ungaro RC et al performed a multicenter retrospective cohort study, and reported that patients with autoimmune and chronic inflammation disease (AICID) are not at increased risk of severe COVID-19 with the exception of those on steroids, and the authors suggested that the AICID patients should continue on biologic and nonbiologic immunosuppression but limit steroid during the COVID-19 pandemic. ${ }^{3}$ Anti-cytokines are suggested as potential treatments for COVID-19, as they may reduce the exaggerated immune response in severe disease. In contrary, steroids have the potential to increase the risk of severe COVID-19. Treatment with steroids have been linked to delayed SARS-CoV-2 viral clearance in COVID-19 patients. Therefore, these findings raise the suggestion to reduce the chronic dose of steroids as low as possible, taking into account the risk-benefit ratio of steroids on immunosuppressive patients. The further study is certainly needed to determine the individual impact of immunosuppressive agent on the course of COVID-19 disease.

1. Center for Systems Science and Engineering (CSSE) at Johns Hopkins University (JHU). COVID-19 Dashboard. Retrieved September 7, 2021, from https://www.arcgis.com/apps/dashboards/bda7594740fd40299423467b48e9ecf6

2. World Health Organization. Spain: WHO Coronavirus disease (COVID-19) Dashboard. Retrieved September 7, 2021, from https://covid19.who.int/region/euro/country/es

3. Ungaro, R.C., Agrawal, M., Park, S., Hirten, R., Colombel, J.-F., Twyman, K., Gulko, P.S. and Klang, E. (2021), Autoimmune and Chronic Inflammatory Disease Patients with COVID-19. ACR Open Rheumatology, 3: 111-115. https://doi.org/10.1002/acr2.11221 